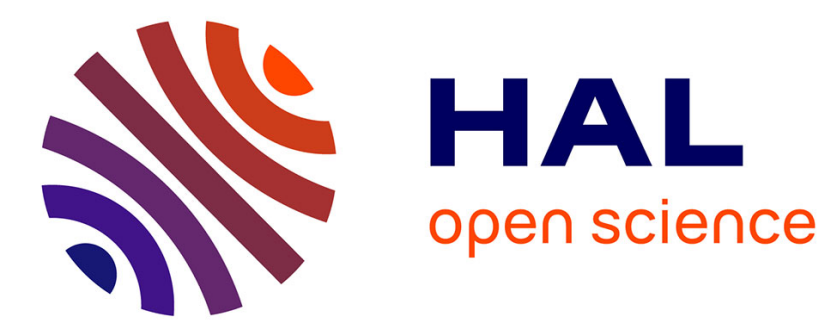

\title{
Near Infrared Fluorogenic Probe as a Prodrug Model for Evaluating Cargo Release by Nanoemulsions
}

Sophie Bou, Xinyue Wang, Nicolas Anton, Andrey S Klymchenko, Mayeul Collot

\section{- To cite this version:}

Sophie Bou, Xinyue Wang, Nicolas Anton, Andrey S Klymchenko, Mayeul Collot. Near Infrared Fluorogenic Probe as a Prodrug Model for Evaluating Cargo Release by Nanoemulsions. Journal of Materials Chemistry B: Materials for Biology and Medicine, 2020, 8 (27), pp.5938-5944. 10.1039/d0tb00783h . hal-03022004

\section{HAL Id: hal-03022004 https://hal.science/hal-03022004}

Submitted on 24 Nov 2020

HAL is a multi-disciplinary open access archive for the deposit and dissemination of scientific research documents, whether they are published or not. The documents may come from teaching and research institutions in France or abroad, or from public or private research centers.
L'archive ouverte pluridisciplinaire HAL, est destinée au dépôt et à la diffusion de documents scientifiques de niveau recherche, publiés ou non, émanant des établissements d'enseignement et de recherche français ou étrangers, des laboratoires publics ou privés. 


\title{
Near Infrared Fluorogenic Probe as a Prodrug Model for Evaluating Cargo Release by Nanoemulsions
}

Received 00th January 20xx, Accepted 00th January 20xx DOI: $10.1039 / \times 0 \times x 00000 x$

\author{
Sophie Bou, ${ }^{a}$ Xinyue Wang, ${ }^{\text {b }}$ Nicolas Anton, ${ }^{\mathrm{b}}$ Andrey S. Klymchenko, ${ }^{\text {a }}$ Mayeul Collot ${ }^{\mathrm{a}}$
}

Nanoemulsions (NEs) are biocompatible and stealth nanodroplets that can efficiently encapsulate hydrophobic cytoactive drugs in their oily core. NEs were shown to accumulate in tumors by enhanced permeability and retention (EPR) effect and thus display appealing features as nanocarriers to selectively deliver drugs to the tumors. However, to ensure efficient encapsulation with minimal early release, drugs must possess a high degree of lipophilicity. To circumvent this limitation, the latter could be transformed into prodrugs with enhanced hydrophobicity. In return, once delivered in the cell, the prodrug must be efficiently transformed into its active drug form. Herein we chemically and reversibly modified a near infrared Huda dye (HD) into pro-fluorophore (Pro-HD), a non-fluorescent and lipophilic prodrug model that was efficiently loaded in NEs. Thanks to the fluorogenecity of the system (fluorescence enhancement of 35 -fold at $723 \mathrm{~nm}$ ), we demonstrated that Pro-HD did not leak out of NEs, was efficiently delivered into cancer cells and was transformed in cellulo into HD. This proof of concept demonstrates the high potential of lipophilic "pro-fluorophore" approach for visualizing delivery of cargos using NEs as nanocarriers.

\section{Introduction}

Over the last 20 years nanoparticles (NPs) have drawn a particular attention in medicine, especially in the field of cancers.1, 2 Indeed, NPs can improve three different aspects of cancer therapy: The tumor imaging, the diagnosis and the treatment. 3, 4, 5, 6, 7 The treatment of tumors can be achieved by specific drug delivery targeted by nanoparticles, ${ }^{8}$ including polymeric NPs, ${ }^{9}, 10$ inorganic NPs, ${ }^{11}$ hydrogels, ${ }^{12}$ lipid polymer hybrid NPs, ${ }^{13}, 14,15$ liposomes $^{16}$ and solid lipid nanoparticles, ${ }^{17}$ thus avoiding side effects of the conventional chemotherapy. Although inorganic NPs (e.g. Quantum dots, iron oxide or gold NPs) and organic NPs (e.g. liposomes, polymeric NPs) can specifically target tumors by vectorization using ligands like $\mathrm{RGD}^{18}$ or folic acid, ${ }^{19,} 20$ their drug loading remains overall limited. ${ }^{21}$ Among NPs, nanoemulsions (NEs) that are composed of an oily core and a surfactant shell (see Fig. 1 A) display attractive features: First, their lipid core enables the encapsulation of an impressive quantity of lipophilic cargos. Then NEs are stealth and constituted of biocompatible components that can be injected in the bloodstream. Finally, their preparation, based on spontaneous emulsification, is readily accessible. ${ }^{22}, 23,24$ Unlike polymeric NPs, the functionalization of NEs by specific ligands for vectorization remains difficult and is still limited to the cellular level. 25, 26, 27, 28 However, it was recently shown using in vivo fluorescence imaging that, when injected in mice, NEs spontaneously

\footnotetext{
a. Laboratory of Biophotonic and Pathologies, CNRS UMR 7021, Université de Strasbourg, Faculté de Pharmacie, 74, Route du Rhin, 67401 Illkirch, France.

b. Université de Strasbourg, CNRS, CAMB UMR 7199, F-67000 Strasbourg, France

* Corresponding author: mayeul.collot@unistra.fr
}

accumulated in tumors by enhanced permeability and retention (EPR) effect. ${ }^{29}, 30$ This phenomenon was observed for solid tumors and it was shown that molecules/particles tend to preferentially accumulate in tumor tissues allowing a faster growth of the tumor compared to other tissues. Consequently, NEs present appealing features to selectively deliver drugs to the tumors. However, loading NEs with cytoactive drugs requires the latter to be solubilized in the oily core and therefore is limited to highly lipophilic drugs. Previously, it was shown that Nile Red, a model of lipophilic drugs, should be modified with lipophilic groups to ensure efficient loading into NPs and to prevent its rapid leakage into biological media. ${ }^{31}$ Unfortunately, most of the drugs do not display a sufficient lipophilic nature to fulfill this requirement. Indeed, common drugs used for chemotherapy medication to treat cancers including: docetaxel, bleomycin, vincristine, prednisolone or doxorubicin, possess several free polar hydroxyl groups. In order to circumvent this issue, a prodrug with a significantly enhanced hydrophobicity compared to its corresponding active drug could be used to be efficiently loaded in NEs. ${ }^{32,} 33$ In return, this chemical modification has to be reversible once the cell target reached in order to release the active drug (Fig. 1A). ${ }^{34}$ In order to enhance the lipophilicity of the drugs, the abovementioned hydroxyl functions can be used to introduce a hydrophobic vector. Carboxylic acids and alcohol functions are readily chemically transformed into esters that can be hydrolyzed in cellulo by specific enzymes called esterases. ${ }^{35}$ It is noteworthy that this strategy is actually used for decades since the most famous drug, namely aspirin is the acetylated prodrug of salicylic acid. ${ }^{36}$ 
A

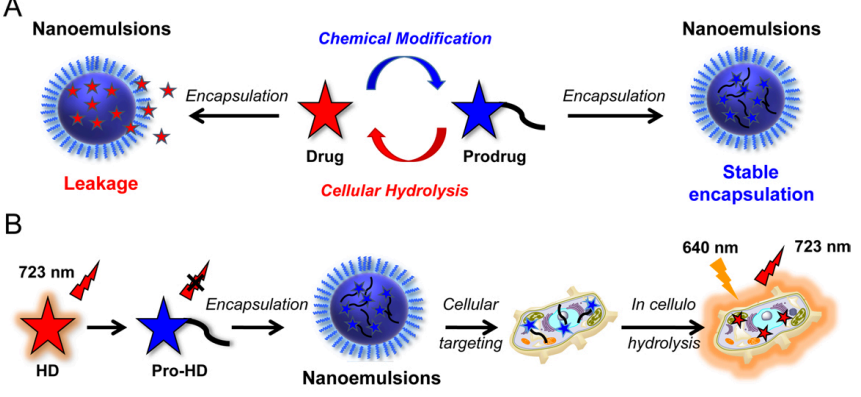

Figure 1. Schematic principle of a prodrug for enhanced encapsulation in NEs (A) and of the developed HD/Pro-HD system as a model to monitor the drug delivery by NEs (B).

Although acetylation is a widely used prodrug strategy, it is not adapted for encapsulation in NEs due to the weak lipophilicity brought by the acetate group. Conversely, caprylic acid is a hydrophobic $C_{8}$ fatty acid, naturally present in mammals' milk and coconut oil. Moreover, unlike esters with longer chain, caprylic esters have been shown to be readily hydrolyzed by lipases and esterases. ${ }^{35}$ Consequently, esterification of drugs into caprylic esters could be used to transform drugs into prodrugs with enhanced lipophilicity. In order to confirm this hypothesis, we herein developed Pro-HD, a fluorogenic drug model based on the esterification of a fluorescent probe (Fig. 1B). The esterified HD called Pro-HD is virtually non-fluorescent and upon hydrolysis releases HD that provokes a fluorescence enhancement of 35-fold in the near-infrared thus making it an efficient system to evaluate the cellular delivery of prodrug by NEs.

\section{Results and discussion}

\section{Design and principle}

In this work we aimed at developing a pro-fluorophore as a prodrug model in order to study by fluorescence imaging its ability to be efficiently delivered by NEs as biocompatible nanocarriers. In addition to its ability to be transformed into the corresponding drug, the encapsulated prodrug should possess two main features: 1 ) It has to be soluble in oil to be efficiently carried by NEs. 2) It should not leak out of the NE before reaching its cellular target (Fig. 1A). ${ }^{31}$ Indeed, if leakage occurs, the uncontrolled release of the cytoactive molecule can lead to negative side effects on healthy cells. Our work relies on the chemical transformation of an emissive fluorescent dye into a hydrophobic and non-emissive pro-dye. The latter bears a hydrophobic vector that quenches or decreases the brightness. After delivery of the pro-fluorophore into the cells, the vector must be cleavable by a cellular process to reveal the fluorescence of the corresponding dye (Fig. 1B). This last step implies that the vector must be biocompatible, avoiding side effects after its cleavage. Dihydroxyxanthene hemicyanines also referred to "Huda dyes" (HD) ${ }^{37}$ are recently developed fluorophores, featuring high brightness and narrow emission band in the near-infrared region (NIR). ${ }^{38}$ Due to these interesting features these fluorophores served to develop efficient probes for hypoxia, ${ }^{39}$ hydrazine, ${ }^{40} \mathrm{pH}^{41,}{ }^{42}$ calcium $^{43}$ and enzyme activity. ${ }^{44}$ Moreover, their spectral properties, including emission in the NIR, make HD probes suitable for in vivo imaging. ${ }^{39,45}$ For these reasons we intended to synthesize a fluorogenic probe based on HD that will display a high degree of hydrophobicity and that could be reversibly turned off by esterification (Scheme 1). To this aim, we designed Pro-HD that possesses several features: 1) The indoleninium moiety bears an octadecyl alkyl chains to maximize the hydrophobicity. 2) The phenolic moiety bears a chlorine atom at the ortho position to lower the pKa of the probe as shown previously. ${ }^{41,} 46$ The addition of the chlorine atom leads to a probe with a reported pKa of 6.2, thus ensuring formation of the brighter phenolate form of HD when exposed to the cytosol.41 3) The hydroxyl group was esterified into a caprylic ester as a lipophilic, biocompatible and cleavable vector. ${ }^{35}$ 4) Finally, small hydrophobic counterion iodide was replaced with bulky hydrophobic counterion of tetraphenylborate family $\left(F_{5}-T P B\right)$, which is important to further increase lipophilicity of cationic dyes. ${ }^{47}$

\section{Synthesis of the probe}

A common access to HD dyes consists in condensing an electron rich phenol on a carbocyanine 7-Cl. ${ }^{38}$ Herein, $\mathrm{Cy} 7-\mathrm{Cl}$ bearing two octadecane chains was prepared (See $\mathrm{SI}$ ) before being reacted with chlororesorcinol to obtain the NIR emitting HD. Then, caprylic acid was used to esterify HD into Pro-HD-I. Finally, the iodine counter anion of Pro-HD-I- was easily exchanged for $\mathrm{F}_{5}-$ TPB (Scheme 1). This last step is further detailed in the section: encapsulation in NEs (see below).

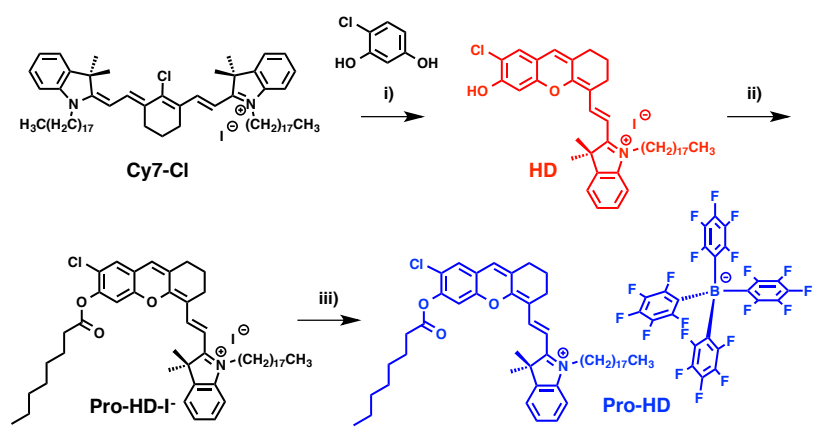

Scheme 1. Synthesis of HD and pro-HD. i) $\mathrm{NEt}_{3}, \mathrm{DMF}, 75^{\circ} \mathrm{C}, 2 \mathrm{~h}$ ii) Caprylic acid, EDC, DMAP, DCM, RT, overnight iii) $F_{5}-T P B-L i, D C M, R T, 5$ min.

\section{Spectroscopic studies}

In order to evaluate the performance of our system, spectroscopic studies of Pro-HD and HD were conducted. Due to the highly hydrophobic nature of HD and Pro-HD, spectroscopic studies could not be performed in aqueous solutions where aggregation occurred. Consequently, methanol was chosen as a polar and protic organic solvent that will mimic a cellular environment where these amphiphilic molecules are expected to end up, namely at the interface between biomembranes and cytosol. Oil (Middle Chain Triglycerides, $\mathrm{MCT}$ ) was also used to study the properties of Pro-HD as it is its solvent when encapsulated in NEs. 

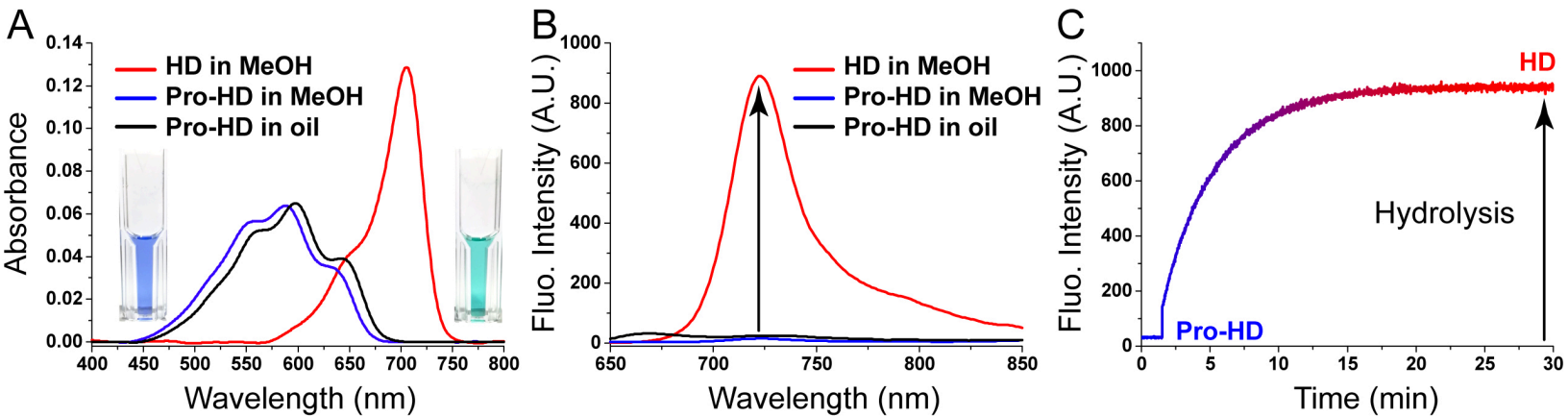

Figure 2. Spectroscopic studies of Pro-HD and HD (1 $\mu \mathrm{M})$. Absorption (A) and emission (B) spectra of HD in methanol and Pro-HD in oil (MCT) and in methanol. (C) In situ hydrolysis of Pro-HD to $\mathrm{HD}(1 \mu \mathrm{M}$ in $\mathrm{MeOH}$ ), evolution of the fluorescence intensity at $723 \mathrm{~nm}$ over $30 \mathrm{~min}$ and after addition of aqueous $\mathrm{NaOH}$ (at $90 \mathrm{~s}, 5 \mu \mathrm{L}$ added in $1 \mathrm{~mL}$, final $\mathrm{NaOH}$ concentration was $250 \mu \mathrm{M}$ ). Excitation wavelength was $640 \mathrm{~nm}$. Pictures of cuvettes are Pro-HD (left) and HD (right) in methanol.

First of all, the results showed that Pro-HD displayed similar properties in methanol and oil (Fig. 2A, B) with broad multipeaks spectra with $\lambda_{\text {Abs max }}$ at 585 and $595 \mathrm{~nm}$, respectively with identical extinction coefficient $\left(\varepsilon=64,000 \mathrm{M}^{-1} . \mathrm{cm}^{-1}\right)$ denoting similar solubility in both solvents at this concentration. Conversely to Pro-HD, HD displayed typical cyanine-shaped absorption spectra with $\lambda_{\text {Abs max }}$ at $705 \mathrm{~nm}$ and a high extinction coefficient value $\left(\varepsilon=127,000 \mathrm{M}^{-1} \cdot \mathrm{cm}^{-1}\right)$ (Fig. 2A). Fluorescence spectroscopy showed that Pro-HD was found to be virtually non-fluorescent in methanol and oil $(\phi=0.006)$, whereas HD emits in the NIR (723 nm) with a quantum yield of 0.2 (Fig. 2B). Interestingly, when excited at $640 \mathrm{~nm}$, which is a common excitation wavelength in bioimaging, the fluorescence enhancement between Pro-HD and HD was 35-fold (Fig. 2B), making Pro-HD a promising fluorogenic probe to evaluate its hydrolysis by the cell machinery. In order to demonstrate the possible hydrolysis of Pro-HD towards $\mathrm{HD}$, a base $(\mathrm{NaOH})$ was added in the methanolic solution. The fluorescence intensity at $723 \mathrm{~nm}$ was monitored over the time (Fig. 2C) and showed that, in these conditions, a plateau was reached after $\sim 15 \mathrm{~min}$. It corresponded to a fluorescence enhancement of 32-fold which is in line with that between Pro-HD and HD (Fig. 2C). After hydrolysis of Pro-HD, the absorption and emission spectra were recorded and corresponded to those of HD (Fig. S1). As discussed above, a chlorine atom was introduced to the structure of HD to lower its pKa and thus to ensure an emissive specie when exposed to cytosolic environment (pH 7.4). Moreover, a lower pKa would also limit the acidification of HD in acidic environment like in early endosomes $(\mathrm{pH} \sim 6)$. Indeed, in methanol and in the presence of acid, HD was found to be virtually non-emissive with a fluorescence intensity at $723 \mathrm{~nm}$ drastically reduced by 60 -fold. Conversely, in the presence of a base (triethylamine), the fluorescence of HD was not significantly affected. (Fig. S2). In the light of these results, the Pro-HD/HD system was considered as a promising probe for encapsulation in NEs.

\section{Encapsulation in NEs}

Efficient encapsulation of a molecule in NEs requires a high lipophilicity in order to: 1) ensure a high solubility in the oil phase and 2) avoid its leakage out of the NEs. 29, 31, 47, 48 As shown in the synthesis section, the iodine counter-ion of Pro-HD-I- was exchanged to $F_{5}-$ TPB to obtain Pro-HD using TPB- $F_{5}$ Li (Fig. 3A). Indeed, modifying the salt forms of a active pharmaceutical ingredients is a common approach to efficiently modulate their physicochemical and biological properties. ${ }^{49}$ This approach was successfully used for enhancing the encapsulation of a potent antituberculosis drug in poly(lactide) microspheres by replacing the polar sodium counterion ion by a lipophilic tetraheptylammonium one. ${ }^{50}$

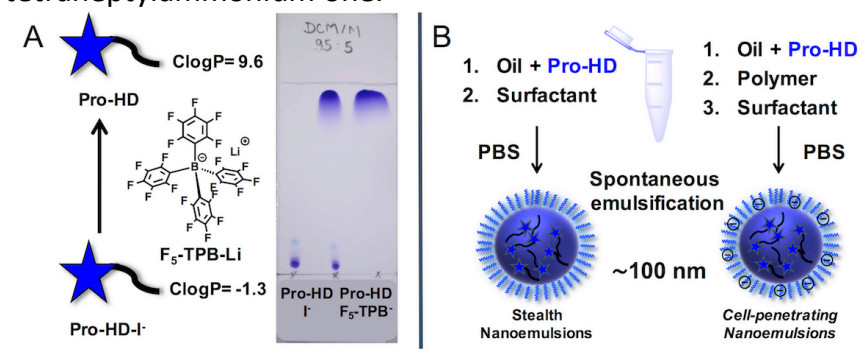

Figure 3. (A) Anion exchange using $F_{5}-T P B$ as a lipophilic counter-ion, TLC (DCM/MeOH, 95:5) depicted the efficiency in decreasing the polarity from Pro-HD.l to Pro-HD ( $F_{5}-\mathrm{TPB}^{-}$ ). (B) Formulation by spontaneous nanoemulsification of Pro-HD loaded stealth NEs (NEs) and cell-penetrating NEs (cPNEs) using poly(maleic anhydride-alt-1-octadecen) (PMAO) as polymer. 25

Although Pro-HD-I- already displayed a hydrophobic structure with a $\mathrm{C}_{18}$ chain combined with a caprylic $\left(C_{8}\right)$ ester, its cationic 
nature combined to the relatively polar iodine counter ion may lead to poor solubility of the dye and potential leakage into biological media. ${ }^{47}$ As shown in figure $3 \mathrm{~A}$, the calculated logP (ClogP) of Pro-HD-I' is drastically increased when transformed in Pro-HD bearing $\mathrm{F}_{5}$-TPB, from -1.3 to 9.6 , respectively. As an illustration of the counterion exchange's effect, the TLC plate is displayed on figure $3 \mathrm{~A}$ and shows the impressive difference of migration distance on silica (from $R_{F} 0.06$ to 0.96 ), in line with earlier reports for other dyes. ${ }^{51}$ After solubilization of Pro-HD in oil at $0.5 \mathrm{wt} \%$ (corresponding to $3.4 \mathrm{mM}$ ), two types of NEs were formulated: 1) Stealth NEs (Fig. 3B), with highly PEGylated surface that prevents from non-specific interactions and thus do not interact with cells. They will be used as control to check whether Pro-HD leaks out of the NEs in the presence of cells. 2) Cell-penetrating NEs, cpNEs (Fig. 3B) that we recently reported, ${ }^{25}$ to deliver the cargo to the cells. The cpNEs are obtained by dissolving poly(maleic anhydride-alt-1-octadecen) (PMAO) in the oil prior to formulation. This alternating polymer is composed of lipophilic carbon chains and cyclic anhydride functions. Upon formulation the latter get hydrolyzed to finally display negative charges at the oil surfactant interface. We reported that the negative charges lead to cell-penetration by endocytosis mediated by non-specific electrostatic interactions. ${ }^{25}$ After formulation, their sizes were measured by DLS and were found to be similar: 97 and $91 \mathrm{~nm}$, respectively (see Fig. S3). Absorption spectra confirmed the efficient encapsulation of Pro-HD in NEs as the optical density of Pro-HD (after correction of the scattering) was similar to those of ProHD in methanol at the same targeted concentration (Fig. S4). Fluorescence spectra, acquired in the same conditions, indicated that Pro-HD was not emissive in both NEs and cpNEs. In order to assess the potential leakage of Pro-HD out of the $\mathrm{NEs}$, the latter were put in the presence of a base, similarly to figure 2C. Indeed, if the Pro-HD leaks out of the NEs in the presence of $\mathrm{NaOH}, \mathrm{HD}$ should be formed and should emit at 723 $\mathrm{nm}$. In these conditions no fluorescence enhancement was obtained for both NEs and cpNEs denoting a strong encapsulation of Pro-HD without any apparent leakage (Fig. S5).
After incubation
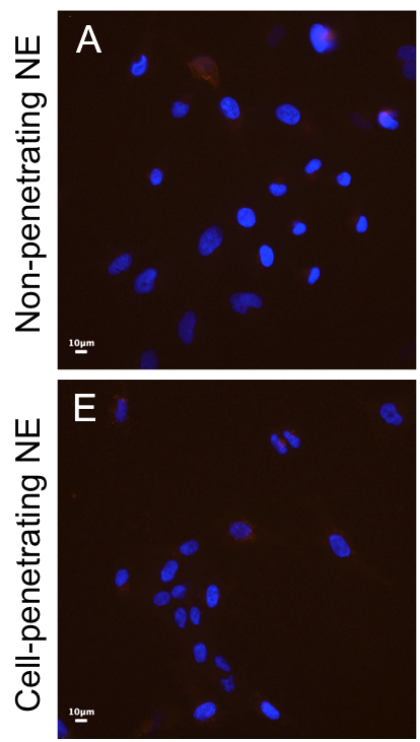

$3 \mathrm{~h}$
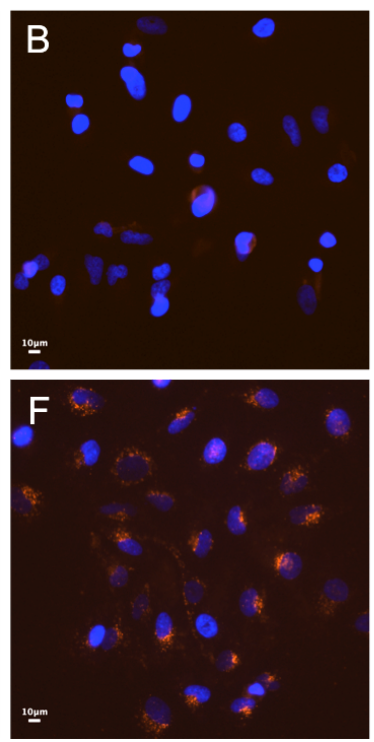

$6 \mathrm{~h}$
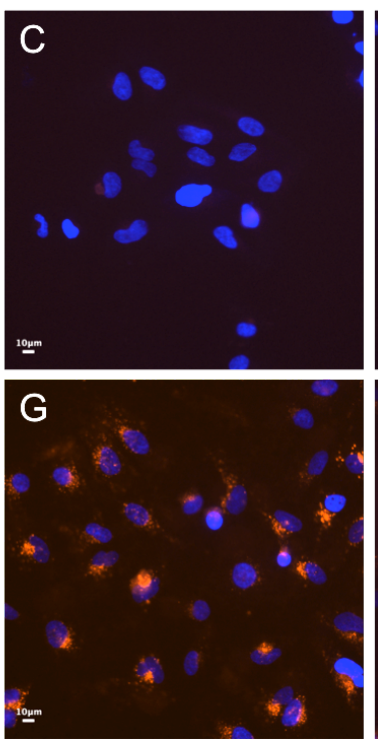

$24 \mathrm{~h}$
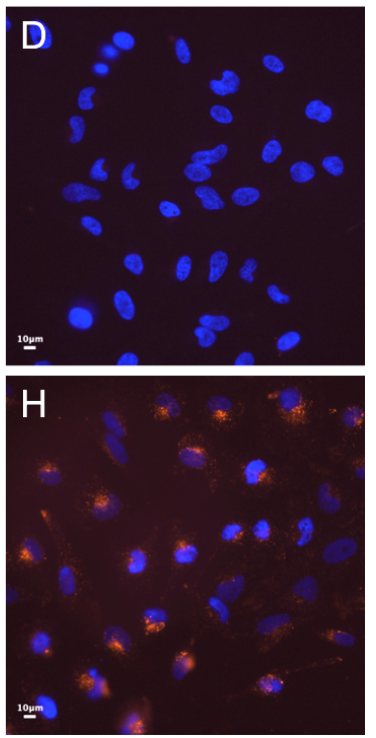

Figure 4. Epi-fluorescence microscopy imaging of fixed HeLa cells pre-incubated for $2 \mathrm{~h}$ in the presence of 0.5 wt\% Pro-HD loaded NEs (top images) and 0.5 wt\% Pro-HD loaded cellpenetrating NEs (bottom images). Final concentration of free Pro-HD and loaded Pro-HD was set to $1 \mu \mathrm{M}$ and the images were acquired in the same conditions of illumination, recording and processing. $\mathrm{HD}$ was excited at $641 \mathrm{~nm}$. The nucleus was stained with Hoechst $\left(5 \mu \mathrm{g} . \mathrm{mL}^{-1}\right)$. Scale bar is $10 \mu \mathrm{m}$.

\section{Evaluation in cellular experiments}

Initially, Pro-HD was incubated for $2 \mathrm{~h}$ in the presence of HeLa cells as a positive control to evaluate the ability of Pro-HD to be hydrolyzed into fluorescent HD in cellulo. Indeed, due to its high hydrophobicity, Pro-HD was expected to spontaneously penetrate the cells in a fast manner. After incubation for $2 \mathrm{~h}$ the cells were washed and presented only a dim signal in the nearinfrared channel. However, upon longer incubation, an increasing fluorescence signal was observed in the cytoplasm of cells, reaching a plateau of intensity after $3 \mathrm{~h}$ (Fig. S6). This control experiment suggested that Pro-HD can be efficiently hydrolyzed in cellulo and that this conversion is not immediate, requiring several hours to occur. In a second time, both Pro-HD
(0.5 wt\%) loaded NEs and cpNEs were incubated in the presence of cells for $2 \mathrm{~h}$ before being washed. Fluorescence imaging showed that whereas cells incubated with stealth NEs displayed no red fluorescence over the time (Fig. 4A-D), those incubated with cell-penetrating NEs showed the progressive appearance of the red fluorescence over the time (Fig. $4 \mathrm{E}-\mathrm{H}$ ). 


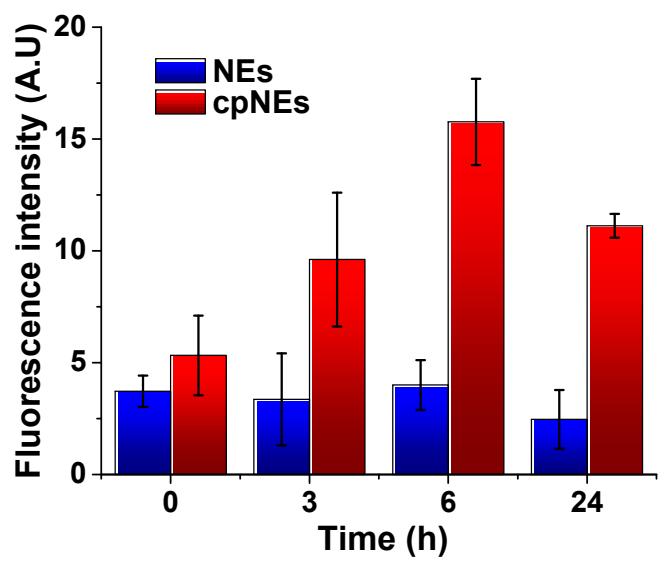

Figure 5. Mean fluorescence intensity of cells at various time points post incubation with NEs and cpNEs. Error bars are the standard deviation between images (10 images per condition, 10 cells per image).

Quantification of fluorescence intensity confirmed these observations and showed that the maximum signal is reached after $6 \mathrm{~h}$ post incubation (Fig. 5). After $24 \mathrm{~h}$ the signal decreased probably due to the repartition of the probe during cell division. This experiment confirmed that Pro-HD is tightly confined in the NEs and does not leak out from the latter ensuring a controlled delivery. It also showed that cpNEs successfully delivered ProHD into cells. It was noteworthy that when Pro-HD was delivered by cpNEs, the fluorescence signal was lower in comparison with direct incubation of Pro-HD and required more time to reach a plateau of fluorescence intensity. Indeed, unlike free Pro-HD, the Pro-HD loaded cpNEs need to: first interact with cells in a non-specific manner and then internalize before being disintegrated to gradually liberate pro-HD within the cell. The summary of our experiments and the mechanism of release is presented in figure S7.

\section{Conclusion}

Visualization of prodrug delivery and release of active drug from nanocarriers is important for better understanding of the bottlenecks in nanomedicine. In particular, it concerns moderately hydrophobic cargos that could be reversibly modified into lipophilic prodrug in order to be loaded in nanoemulsions for controlled drug delivery. Here, we developed Pro-HD, a lipophilic near infrared fluorogenic probe as a model of prodrug to assess the controlled cargo delivery and release by nanoemulsions into cells. For this purpose, HD, our fluorescent drug model, was esterified with the hydrophobic and biocompatible caprylic acid, and its counter ion was exchanged for the hydrophobic $\mathrm{F}_{5}$-TPB giving rise to Pro$H D$, a highly lipophilic fluorogenic prodrug model. We demonstrated that Pro-HD, which is virtually non-fluorescent, could be hydrolyzed into HD in cuvette as well as in cellulo, provoking a fluorescence enhancement of 35 -fold in the near infrared region $\left(\lambda_{E m}=723 \mathrm{~nm}\right)$. Due to its high lipophilicity, ProHD was efficiently loaded into NEs with no leakage thus avoiding undesired premature delivery. Using cell-penetrating NEs, we were able to show that Pro-HD was delivered to cells and transformed into HD within $\sim 6 \mathrm{~h}$. We believe that this proof of concept can lead to the development of efficient prodrugs based on similar modifications of drugs requiring NEs as nanocarriers for efficient administration to target and treat tumors. As perspective, and taking advantage of the near infrared emission of HD, we also believe that this system is amenable to fluorescence in vivo imaging experiments, this will be the subject of a dedicated study.

\section{Experimental}

\section{Materials}

The solvents were of analytical grade and dry, reagent and starting materials were purchased form TCl, Alfa Aesar or Merck Millipor. Labrafac WL ${ }^{\circledR} 1349$ (medium chain triglycerides, MCT) was obtained from Gattefossé (Saint-Priest, France). Kolliphor ELP $^{\circledR}$, was from BASF (Ludwigshafen, Germany). Poly(maleic anhydride-alt-a-octadecene) (PMAO) was purchased from Sigma Aldrich (St. Louis, USA). Phosphate buffered saline (PBS) was from Eurobio (Gourtaboeuf, France) and fetal bovine serum (FBS) from Lonza.

\section{Synthesis}

Protocols and characterizations can be found in the supplementary information. NMR spectra were recorded on a Bruker Avance III $400 \mathrm{MHz}$ spectrometer. Mass spectra were obtained using an Agilent Q-TOF 6520 mass spectrometer.

\section{Spectroscopic studies}

All the solvents were spectro grade. Absorption and emission spectra were recorded on a Cary 400 Scan ultraviolet-visible spectrophotometer (Varian) and a FluoroMax-4 spectrofluorometer (Horiba Jobin Yvon) respectively. For standard recording of fluorescence spectra, the emission was collected $10 \mathrm{~nm}$ after the excitation wavelength. All the spectra were corrected from wavelength-dependent response of the detector. Quantum yields were determined by comparison with Rhodamine 800 in $\mathrm{EtOH}(\mathrm{QY}=0.25)^{52}$ as reference using the following equation:

$$
\mathrm{QY}=\mathrm{QY}_{R} \times \frac{I \times O D_{R} \times n^{2}}{I_{R} \times O D \times n_{R}^{2}}
$$

where $Q Y$ is the quantum yield, I is the integrated fluorescence intensity, $\mathrm{n}$ is the refractive index, and OD is the optical density at the excitation wavelength. $\mathrm{R}$ represents the reference.

\section{Formulation of NEs}

Nano-emulsions (NEs) were formulated with spontaneous emulsification method, as described previously. ${ }^{25}$ In $100 \mathrm{mg}$ of Medium Chain Triglyceride oil (MCT), Pro-HD $(0.5 \mathrm{mg})$ in acetone was added before being heated at $80^{\circ} \mathrm{C}$ until complete evaporation of acetone and solubilization of Pro-HD in MCT. 67 $\mathrm{mg}$ of Kolliphor (surfactant) was then added at $80^{\circ} \mathrm{C}$ and after 
mixing for $10 \mathrm{~min}, 0.25 \mathrm{~mL}$ of pre-heated PBS was added to form nanoemulsions. For cell-penetrating NEs, PMAO (1 mg) was first dissolved in MCT prior to the addition of Pro-HD. The size of the obtained NEs was measured by Dynamic Light Scattering (DLS) using a Malvern Zetasizer Nano ZSP (Malvern, U.K.). For the size measurement, all the emulsions were diluted by 100 times and measured at a temperature of $25^{\circ} \mathrm{C}$. Both size distribution and polydispersity index (PDI) were recorded.

\section{Cellular imaging}

HeLa cells $\left(\right.$ ATCC $^{\circledR}$ CCL- $^{\text {TM }}$ ) were grown in Dulbecco's Modified Eagle Medium without phenol red (DMEM, Gibco-Invitrogen) supplemented with $10 \%$ fetal bovine serum (FBS, Lonza), 1\% LGlutamine (Sigma Aldrich) and $0.1 \%$ antibiotic solution (gentamicin, Sigma-Aldrich) at $37^{\circ} \mathrm{C}$ in humidified atmosphere containing $5 \% \mathrm{CO}_{2}$. Cells were seeded onto a $35 \mathrm{~mm}$ glassbottomed imaging dish (Ibidi ${ }^{\circledR}$ ) at a density of $5 \times 10^{4}$ cells/well $24 \mathrm{~h}$ before incubation experiments. The cells were then washed 3 times with PBS and incubated $2 \mathrm{~h}$ in opti-MEM in the presence of Pro-HD, Pro-HD loaded NEs and Pro-HD loaded cPNEs ( $1 \mu \mathrm{M}$ based on absorbance of Pro-HD) before being washed again 3 times with PBS. The cells were then incubated at various times in Dulbecco's modified Eagle medium (DMEM, Gibco-Invitrogen), supplemented with $10 \%$ fetal bovine serum (FBS, Lonza) and $1 \%$ antibiotic solution (penicillin-streptomycin, Gibco-Invitrogen) at $37^{\circ} \mathrm{C}$ in humidified atmosphere containing $5 \% \mathrm{CO}_{2}$. Prior to imaging, nucleus was stained with Hoechst (5 $\mu \mathrm{g} \cdot \mathrm{mL}^{-1}$ ) and the cells were washed with PBS and fixed with $4 \%$ PFA at room temperature for 5 minutes before being washed 3 times with PBS. The images were acquired with a Nikon Ti-E inverted epi-fluorescence microscope, using CFI Plan Apo $\times 60$ oil (NA = 1.4) or CFI Plan Apo $\times 20$ air (NA = 0.75) objective, and a Hamamatsu Orca Flash 4 sCMOS camera. Hoechst and HD were excited at 395 and $641 \mathrm{~nm}$, respectively, using lightemitting diode (SpectraX, Lumencor). The images were recorded using NIS Elements and then processed with Icy software.

\section{Conflicts of interest}

There are no conflicts to declare

\section{Acknowledgements}

This work was supported by ERC Consolidator grant BrightSens 648528. XW was founded by the China Scholarship Council PhD fellowship (CSC No. 201706240033). We acknowledge Dr. Delphine Garnier and Dr. Estefania Oliva from the Service de Chimie Analytique (SCA) for their assistance in the LC-MS and RMN analyses.

\section{References}

11 J. Shi, P. W. Kantoff, R. Wooster and O. C. Farokhzad, Nat. Rev. Cancer, 2017, 17, 20-37.
22 K. Cho, X. Wang, S. Nie, Z. (Georgia) Chen and D. M. Shin, Clin. Cancer Res., 2008, 14, 1310-1316.

33 M. V. Yigit, A. Moore and Z. Medarova, Pharm. Res., 2012, 29, 1180.

$4 \quad 4$ X. Huang and M. A. El-Sayed, J. Adv. Res., 2010, 1, 13-28.

$5 \quad 5 \quad$ R. A. Revia and M. Zhang, Mater. Today, 2016, 19, 157168.

66 N. Rapoport, Z. Gao and A. Kennedy, JNCl J. Natl. Cancer Inst., 2007, 99, 1095-1106.

77 X.-H. Peng, X. Qian, H. Mao, A. Y. Wang, Z. (Georgia) Chen, S. Nie and D. M. Shin, Int. J. Nanomedicine, 2008, 3, 311.

88 A. H. Faraji and P. Wipf, Bioorg. Med. Chem., 2009, 17, 2950-2962.

99 J. M. Chan, P. M. Valencia, L. Zhang, R. Langer and O. C. Farokhzad, Methods Mol. Biol. Clifton NJ, 2010, 624, 163-175.

1010 K. M. El-Say and H. S. El-Sawy, Int. J. Pharm., 2017, 528, 675-691.

1111 M. Liong, J. Lu, M. Kovochich, T. Xia, S. G. Ruehm, A. E. Nel, F. Tamanoi and J. I. Zink, ACS Nano, 2008, 2, 889.

1212 M. Hamidi, A. Azadi and P. Rafiei, Adv. Drug Deliv. Rev., 2008, 60, 1638-1649.

1313 B. Mandal, H. Bhattacharjee, N. Mittal, H. Sah, P. Balabathula, L. A. Thoma and G. C. Wood, Nanomedicine Nanotechnol. Biol. Med., 2013, 9, 474-491.

1414 K. Hadinoto, A. Sundaresan and W. S. Cheow, Eur. J. Pharm. Biopharm., 2013, 85, 427-443.

1515 L. Zhang, J. M. Chan, F. X. Gu, J.-W. Rhee, A. Z. Wang, A. F. Radovic-Moreno, F. Alexis, R. Langer and O. C. Farokhzad, ACS Nano, 2008, 2, 1696.

1616 V. P. Torchilin, Nat. Rev. Drug Discov., 2005, 4, 145-160.

1717 W. Mehnert and K. Mäder, Adv. Drug Deliv. Rev., 2001, 47, 165-196.

1818 H.-Y. Lee, Z. Li, K. Chen, A. R. Hsu, C. Xu, J. Xie, S. Sun and X. Chen, J. Nucl. Med., 2008, 49, 1371-1379.

1919 S. Mohapatra, S. K. Mallick, T. K. Maiti, S. K. Ghosh and P. Pramanik, Nanotechnology, 2007, 18, 385102.

2020 Z. Zhang, J. Jia, Y. Lai, Y. Ma, J. Weng and L. Sun, Bioorg. Med. Chem., 2010, 18, 5528-5534.

2121 C. Yan, Z. Guo, Y. Shen, Y. Chen, H. Tian and W.-H. Zhu, Chem. Sci., 2018, 9, 4959-4969.

2222 N. Anton and T. F. Vandamme, Int. J. Pharm., 2009, 377, 142-147.

2323 D. J. McClements, Soft Matter, 2012, 8, 1719-1729.

2424 M. Yao, H. Xiao and D. J. McClements, Annu. Rev. Food Sci. Technol., 2014, 5, 53-81.

2525 M. F. Attia, S. M. Dieng, M. Collot, A. S. Klymchenko, C. Bouillot, C. A. Serra, M. Schmutz, M. Er-Rafik, T. F. Vandamme and N. Anton, Macromol. Biosci., 2017, 17, 1600471.

2626 M. F. Attia, N. Anton, R. Bouchaala, P. Didier, Y. Arntz, N. Messaddeq, A. S. Klymchenko, Y. Mély and T. F. Vandamme, RSC Adv., 2015, 5, 74353-74361.

2727 D. A. Estabrook, A. F. Ennis, R. A. Day and E. M. Sletten, Chem. Sci., 2019, 10, 3994-4003.

2828 A. Saito, S. Yamamoto, R. Ochi, K. Inoue, S. Hadano, S. Watanabe, T. Nakayama and Y. Niko, Bull. Chem. Soc. Jpn., , DOI:10.1246/bcsj.20200014.

2929 R. Bouchaala, L. Mercier, B. Andreiuk, Y. Mély, T. Vandamme, N. Anton, J. G. Goetz and A. S. Klymchenko, J. Controlled Release, 2016, 236, 57-67.

3030 M. A. Radicchi, J. V. de Oliveira, A. C. P. Mendes, D. M. de Oliveira, L. A. Muehlmann, P. C. Morais, R. B. Azevedo and J. P. F. Longo, J. Mater. Chem. B, 2018, 6, 7306-7316.

3131 A. S. Klymchenko, E. Roger, N. Anton, H. Anton, I. Shulov, J. Vermot, Y. Mely and T. F. Vandamme, RSC Adv., 2012, 2, 11876-11886.

3232 A. L. Dos Santos Câmara, G. Nagel, H. R. Tschiche, C. M. Cardador, L. A. Muehlmann, D. M. de Oliveira, P. Q. Alvim, R. 
B. Azevedo, M. Calderón and J. P. Figueiró Longo, Nanomed., 2017, 12, 1751-1765.

3333 J.-J. Wang, K. C. Sung, O. Y.-P. Hu, C.-H. Yeh and J.-Y. Fang, J. Controlled Release, 2006, 115, 140-149.

3434 R. K. Keservani and A. K. Sharma, Nanoconjugate Nanocarriers for Drug Delivery, CRC Press, 2018.

3535 T. D. Nalder, T. D. Ashton, F. M. Pfeffer, S. N. Marshall and C. J. Barrow, Biochimie, 2016, 128-129, 127-132.

3636 H. Dreser, Pflugers Arch 189976306-18.

3737 L. Yuan, W. Lin, K. Zheng, L. He and W. Huang, Chem. Soc. Rev., 2012, 42, 622-661.

3838 L. Yuan, W. Lin, S. Zhao, W. Gao, B. Chen, L. He and S. Zhu, J. Am. Chem. Soc., 2012, 134, 13510-13523.

3939 Y. Liu, L. Teng, L. Chen, H. Ma, H.-W. Liu and X.-B. Zhang, Chem. Sci., 2018, 9, 5347-5353.

4040 S. Zhu, W. Lin and L. Yuan, Anal. Methods, 2013, 5, 34503453.

4141 Y. Li, Y. Wang, S. Yang, Y. Zhao, L. Yuan, J. Zheng and R. Yang, Anal. Chem., 2015, 87, 2495-2503.

4242 Q. Wan, S. Chen, W. Shi, L. Li and H. Ma, Angew. Chem. Int. Ed., 2014, 53, 10916-10920.

4343 M. Collot, F. Ponsot and A. S. Klymchenko, Chem. Commun., 2017, 53, 6117-6120.
4444 Z. Luo, L. Feng, R. An, G. Duan, R. Yan, H. Shi, J. He, Z. Zhou, C. Ji, H.-Y. Chen and D. Ye, Chem. - Eur. J., 2017, 23, 1477814785.

4545 H. Chen, W. Lin, H. Cui and W. Jiang, Chem. - Eur. J., 2015, 21, 733-745.

4646 G. Despras, A. I. Zamaleeva, L. Dardevet, C. Tisseyre, J. G. Magalhaes, C. Garner, M. D. Waard, S. Amigorena, A. Feltz, J.M. Mallet and M. Collot, Chem. Sci., 2015, 6, 5928-5937.

4747 V. N. Kilin, H. Anton, N. Anton, E. Steed, J. Vermot, T. F. Vandamme, Y. Mely and A. S. Klymchenko, Biomaterials, 2014, 35, 4950-4957.

4848 X. Wang, N. Anton, P. Ashokkumar, H. Anton, T. K. Fam, T. Vandamme, A. S. Klymchenko and M. Collot, ACS Appl. Mater. Interfaces, 2019, 11, 13079-13090.

4949 D. Gupta, D. Bhatia, V. Dave, V. Sutariya and S. Varghese Gupta, Molecules, 2018, 23, 1719.

$5050 \mathrm{H}$. Zhou, C. Lengsfeld, D. J. Claffey, J. A. Ruth, B. Hybertson, T. W. Randolph, K. Ng and M. C. Manning, J. Pharm. Sci., 2002, 91, 1502-1511.

5151 B. Andreiuk, A. Reisch, E. Bernhardt and A. S. Klymchenko, Chem. - Asian J., 2019, 14, 836-846.

5252 A. Alessi, M. Salvalaggio and G. Ruzzon, J. Lumin., 2013, 53 134, 385-389.

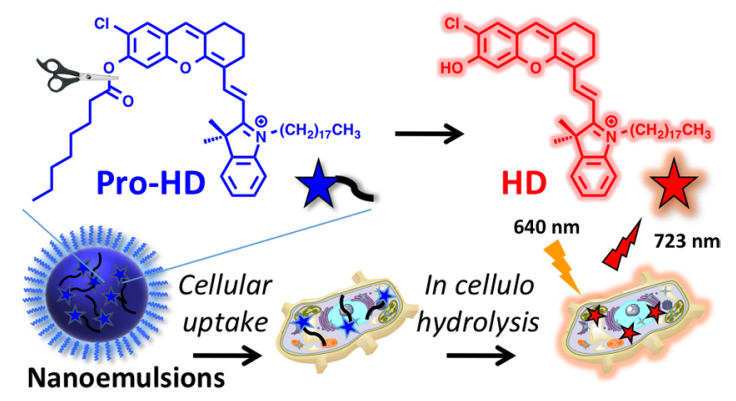

Graphical Abstract 